\title{
Motion direction discrimination training reduces perceived motion repulsion
}

\author{
Ke Jia ${ }^{1,2,3} \cdot$ Sheng $\mathrm{Li}^{1,2,3}$
}

Published online: 27 December 2016

(C) The Psychonomic Society, Inc. 2016

\begin{abstract}
Participants often exaggerate the perceived angular separation between two simultaneously presented motion stimuli, which is referred to as motion repulsion. The overestimation helps participants differentiate between the two superimposed motion directions, yet it causes the impairment of direction perception. Since direction perception can be refined through perceptual training, we here attempted to investigate whether the training of a direction discrimination task changes the amount of motion repulsion. Our results showed a direction-specific learning effect, which was accompanied by a reduced amount of motion repulsion both for the trained and the untrained directions. The reduction of the motion repulsion disappeared when the participants were trained on a luminance discrimination task (control experiment 1 ) or a speed discrimination task (control experiment 2), ruling out any possible interpretation in terms of adaptation or training-induced attentional bias. Furthermore, training with a direction discrimination task along a direction $150^{\circ}$ away from both directions in the transparent stimulus (control experiment 3 ) also had little effect on the amount of motion repulsion, ruling out the contribution of task learning. The changed motion repulsion observed in the main experiment was consistent with the prediction of the recurrent model of perceptual learning.
\end{abstract}

Sheng Li

sli@pku.edu.cn

1 Department of Psychology and Beijing Key Laboratory of Behavior and Mental Health, Peking University, 5 Yiheyuan Road, Haidian, Beijing 100871, China

2 PKU-IDG/McGovern Institute for Brain Research, Peking University, 5 Yiheyuan Road, Haidian, Beijing 100871, China

3 Key Laboratory of Machine Perception (Ministry of Education), Peking University, 5 Yiheyuan Road, Haidian, Beijing 100871, China
Therefore, our findings demonstrate that training in direction discrimination can benefit the precise direction perception of the transparent stimulus and provide new evidence for the recurrent model of perceptual learning.

Keywords Perceptual learning $\cdot$ Visual perception $\cdot$ Motion

\section{Introduction}

Precise direction perception of a moving object is important for primates to survive. It helps predators catch their prey and keeps pedestrians from being hit by upcoming vehicles. In the laboratory, the human sensitivity of direction perception as measured by a motion direction discrimination task is approximately $5^{\circ}$ (Ball \& Sekuler, 1987; Bruyn \& Orban, 1988). However, when the stimulus consists of two superimposed components moving along two different directions, the participants show a strong tendency to exaggerate their perceived angular separation (Hiris \& Blake, 1996; Marshak \& Sekuler, 1979; Rauber \& Treue, 1999; Wilson \& Kim, 1994). This well-known motion illusion is referred to as motion repulsion and has been interpreted as evidence of the mutual inhibition between the direction-tuned neurons in the motion-selective visual areas (Blakemore \& Carpenter, 1970; Chen, Meng, Matthews, \& Qian, 2005; Hiris \& Blake, 1996; Kim \& Wilson 1996; Marshak \& Sekuler, 1979; Rauber \& Treue, 1999; Wilson \& Kim, 1994) (see Fig. 1 for a description of the model). The extent of the overestimation has been reported to be up to $20^{\circ}$ (Marshak \& Sekuler, 1979) and remains approximately $10^{\circ}$ even after the exclusion of reference repulsion (Rauber \& Treue, 1999). Although this bias in direction estimation may be helpful in differentiating between two simultaneously presented motion components, it impairs the fine perception of direction. 

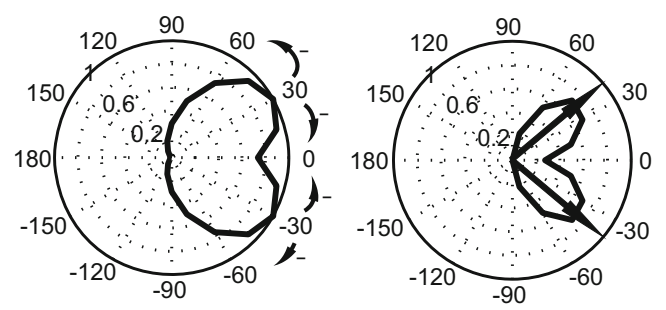

Fig. 1 Mutual inhibition model of motion repulsion (Wilson \& Kim, 1994). Left panel: The initial responses of the direction-selective neurons to the transparent motion stimulus (i.e., $\pm 30^{\circ}$ ) are equal to their maximum responses to its component directions. These neurons then dynamically inhibit each other with larger inhibition coefficients to the adjacent neurons. The extent of the inhibition is proportional to the initial response of the neurons. Right panel: The equilibrium state after mutual inhibition. The arrows point to the perceived directions (defined as the vector summations of the activity of all the neurons) that shift away from the true directions of the two stimuli

The sensitivity of fine direction perception can be enhanced through extensive training (Ball \& Sekuler, 1987), which is referred to as perceptual learning (Gilbert, Sigman, \& Crist, 2001; Sagi \& Tanne, 1994; Shibata, Sagi, \& Watanabe, 2014). Importantly, the reported training effects were highly specific to the trained location, feature, or eye (Ahissar \& Hochstein, 1997; Ball \& Sekuler, 1987; Crist, Kapadia, Westheimer, \& Gilbert, 1997; Fahle, 1997; Fahle \& Morgan, 1996; Fiorentini \& Berardi, 1980; Jehee et al., 2012), indicating concurrent plastic changes in the sensory areas, which is also supported by evidence from physiological and neuroimaging studies (Hua et al., 2010; Jehee et al., 2012; Schoups, Vogels, Qian, \& Orban, 2001; Shibata et al., 2012). A computational model of discrimination learning further proposed that the plastic changes in the sensory areas are produced by the change in the inhibitory interaction between the direction-tuned neurons (Teich \& Qian, 2003). Moreover, perceptual learning was also accompanied by task learning (Watanabe \& Sasaki, 2015), modification of the decision-making process (Dosher, Jeter, Liu, \& Lu, 2013; Dosher \& Lu, 1998, 1999; Law \& Gold, 2008, 2009), and enhanced attentional gain (Byers \& Serences, 2014).

Although the training effects have been demonstrated across sensory modalities (Recanzone, Schreiner, \& Merzenich, 1993; Recanzone, Merzenich, Jenkins, Grajski, \& Dinse, 1992; Recanzone, Merzenich, \& Schreiner, 1992) and perceptual tasks (Ball \& Sekuler, 1987; Fine \& Jacobs, 2002; Fiorentini \& Berardi, 1980; Jehee et al., 2012; Karni \& Sagi, 1991), whether perceptual learning could modify fine direction perception with a transparent stimulus has not yet been investigated. Given that perceptual learning is accompanied by plastic changes in the sensory areas and attentional process, both of which affect the mutual inhibition between the direction-tuned neurons (Chen et al., 2005; Kim \& Wilson 1996), it is reasonable to expect training-induced changes in the motion repulsion. Here, we directly tested this hypothesis by training participants to perform a direction discrimination task along one of the directions of the transparent stimulus. If the training modified the amount of motion repulsion, we would further investigate the cause of the observed behavioral effect using control experiments.

In brief, our results showed a reduced amount of motion repulsion along both the trained and the untrained directions. By combining the mutual inhibition model of motion repulsion and the recurrent model of perceptual learning, we can make a clear prediction of the change in the motion repulsion along the untrained direction, which was consistent with our behavioral results. The amount of motion repulsion remained unchanged when the participants were trained with the luminance discrimination task or the speed discrimination task, suggesting that the change in the amount of motion repulsion cannot be attributed to adaptation or training-induced attentional bias. In addition, training participants with the direction discrimination task along a direction $150^{\circ}$ away from both directions in the transparent stimulus had little effect on the amount of motion repulsion, ruling out the contribution of task learning. Therefore, our findings suggest that training in direction discrimination can benefit the precise direction perception of the transparent stimulus, and this refinement may stem from the modified recurrent process in the sensory areas.

\section{Methods}

\section{Subjects}

The study consisted of a main experiment (16 participants, nine females, age range: 18-25 years) and three control experiments (control experiment 1: luminance discrimination training, 16 participants, nine females, age range: 18-24 years; control experiment 2: speed discrimination training, 16 participants, nine females, age range: 19-27 years; control experiment 3: direction discrimination training, eight participants, three females, age range: 20-27 years). All participants had normal or corrected-to-normal vision and were naïve to the purpose of the experiment. All participants gave written informed consent, and the study was approved by the local ethics committee. The work was carried out in accordance with the Code of Ethics of the World Medical Association (Declaration of Helsinki).

\section{Apparatus}

All stimuli (dynamic random dot displays, DRDs) were displayed on a CRT screen (resolution: $1,024 \times 768$, refresh rate: $120 \mathrm{~Hz}$ ) and viewed binocularly at a distance of $60 \mathrm{~cm}$. A chin rest was used to stabilize the participants' heads. All experiments were implemented using Psychtoolbox 3.0 
(Brainard, 1997; Pelli, 1997) in MATLAB 2009b (The MathWorks, Natick, MA, USA) and conducted in a dimly lit room. Participants used a computer keyboard to make their responses.

\section{Stimuli}

To generate a DRD, we randomly generated a set of dots, which was presented for one frame and replaced by another set of dots with a constant positional offset (Britten, Shadlen, Newsome, \& Movshon, 1992). All DRDs were presented in an invisible $10^{\circ}$ diameter aperture in the center of a black background $\left(\sim 0 \mathrm{~cd} / \mathrm{m}^{2}\right)$. All dots in the DRDs moved in the same direction. If any of these dots moved out of the aperture, it was wrapped around to reappear from the opposite side to conserve the dot density. The gamma function was used to rectify the luminance of the stimuli. After the gamma correction, the gray-scale values (gsv) were proportional to the luminance of the stimuli. Therefore, we can use gray-scale values to represent the luminance of the stimuli. The range of the gray-scale values was from $0\left(\sim 0 \mathrm{~cd} / \mathrm{m}^{2}\right)$ to 255 $\left(\sim 85.5 \mathrm{~cd} / \mathrm{m}^{2}\right)$.

In this study, we used four different tasks: the direction discrimination task, the luminance discrimination task, the speed discrimination task, and the motion repulsion task. In the first three tasks, each DRD lasted for $500 \mathrm{~ms}$ and consisted of 200 gray dots (gsv: 127, $2.55 \mathrm{dots} / \mathrm{deg}^{2}$ ). In the motion repulsion task, each DRD lasted for 1,000 ms and consisted of 200 gray dots in each direction (gsv: 127, $5.09 \mathrm{dots} / \mathrm{deg}^{2}$ ).

\section{Tasks}

\section{Direction discrimination task}

A typical trial is shown in Fig. 2B. A red circular fixation point was presented at the start of each trial and remained visible throughout the trial. After a 500-ms delay, two DRDs (a reference and a test) were presented successively in two stimulus intervals. Each DRD was shown for $500 \mathrm{~ms}$, with an interstimulus interval of $500 \mathrm{~ms}$. The reference DRD with a fixed direction of $\theta^{\circ}\left(0^{\circ}\right.$ represents the upward direction, and clockwise directions are marked as positive values) was presented randomly in either the first or the second stimulus interval, and the test DRD with a varied motion direction controlled by the methods of constant stimuli in the test sessions or classical staircases in the training sessions was displayed in the other interval. In the test sessions, the stimulus direction was randomly selected from a set of values with equal probability $(\theta-$ $\left.8^{\circ}, \theta-6^{\circ}, \theta-4^{\circ}, \theta-2^{\circ}, \theta^{\circ}, \theta+2^{\circ}, \theta+4^{\circ}, \theta+6^{\circ}, \theta+8^{\circ}\right)$. In the training sessions, the step size of the staircase was $0.5^{\circ}$. The direction range and the step size for the test DRD were determined by a preliminary test using a separate group of naïve participants. The dots in all DRDs moved at a speed of $4 \mathrm{deg} / \mathrm{s}$. The
A

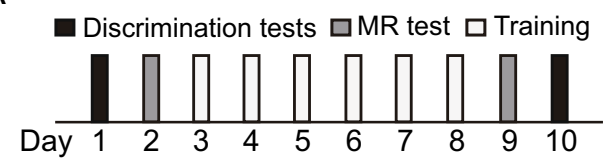

B

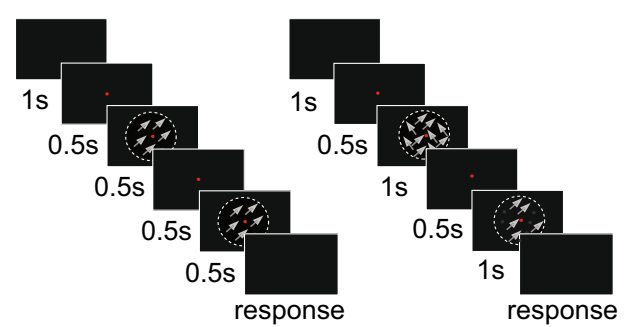

Fig. 2 (A) Experimental procedure of the experiments. (B) A typical trial for the discrimination tasks in the training sessions and test sessions. The participants' task was to report whether the second dynamic random dot display (DRD) was more clockwise or counterclockwise in the direction discrimination task (brighter or dimmer in the luminance discrimination task, faster or slower in the speed discrimination task) compared to the first DRD. (C) A typical trial for the motion repulsion task. The participants' task was to judge whether the direction of the moving dots in the second DRD was clockwise or counterclockwise relative to the corresponding direction in the first DRD. The dimmer dots in the second DRD were static

participants were asked to report whether the motion direction of the DRD in the second stimulus interval was tilted clockwise or counterclockwise relative to the direction of the DRD in the first interval. The luminance of the dots in the direction discrimination task was set to $\varphi$ (in gsv). The inter-trial interval was $1,000 \mathrm{~ms}$.

\section{Luminance discrimination task}

This task was identical to the direction discrimination task with two exceptions. First, the task was to report whether the dots in the second stimulus interval were brighter or dimmer relative to those in the first interval. Second, the direction of the DRDs was fixed at $\theta^{\circ}$; the luminance of the dots in the reference DRD was set to $\varphi$ (gsv), while the luminance of the dots in the test DRD was controlled with the methods of constant stimuli in the test sessions or classical staircases in the training sessions. The stimulus range in the test sessions was $(\varphi-30, \varphi-25, \varphi-20, \varphi-15, \varphi-10, \varphi-5, \varphi, \varphi+5, \varphi+10, \varphi+15$, $\varphi+20, \varphi+25, \varphi+30)$, and the step size in the training sessions was 2 gsv.

\section{Speed discrimination task}

In the speed discrimination task, participants were asked to report whether the dots in the second stimulus interval moved faster or slower relative to those in the first interval. The direction of the DRDs was fixed at $\theta^{\circ}$. The luminance of the dots was set to $\varphi$ (gsv). The speed of the dots in the reference 
DRD was $4 \mathrm{deg} / \mathrm{s}$, while the speed of the dots in the test DRD was controlled by the methods of constant stimuli in the test sessions or classical staircases in the training sessions. The speed range in the test sessions was $(3.10,3.33,3.55,3.78$, $4,4.23,4.45,4.68,4.9)$. The step size in the training sessions was $0.03 \mathrm{deg} / \mathrm{s}$.

\section{Motion repulsion task}

The motion repulsion task differed from the direction discrimination task in three aspects. First, the reference DRD consisted of two sets of dots moving in the directions of $30^{\circ}$ and $-30^{\circ}$ simultaneously and was always presented in the first stimulus interval for $1,000 \mathrm{~ms}$. This arrangement was set to prevent participants from attending to only one of the directions when viewing the reference DRD. Based on our preliminary test, we chose an offset of $60^{\circ}$ and a presentation time of $1,000 \mathrm{~ms}$ to induce a stable and large motion repulsion, which were also consistent with a previous study (Rauber \& Treue, 1999). Second, the test DRD also consisted of two sets of dots. However, one set of dots remained static to conserve the dot density, while the other set moved in the direction of $( \pm(\theta-$ $\left.16^{\circ}\right), \pm\left(\theta-12^{\circ}\right), \pm\left(\theta-8^{\circ}\right), \pm\left(\theta-4^{\circ}\right), \pm \theta^{\circ}, \pm\left(\theta+4^{\circ}\right), \pm\left(\theta+8^{\circ}\right), \pm(\theta+$ $\left.\left.12^{\circ}\right), \pm\left(\theta+16^{\circ}\right)\right)$. Third, participants were asked to report whether the direction of the moving dots in the test DRD tilted clockwise or counterclockwise relative to the corresponding direction in the reference DRD. It should be noted here that previous studies measuring the amount of motion repulsion with an estimation task have shown that even when a single motion direction was presented, the perceived direction would also be repelled by the nearest cardinal axis, which is referred to as reference repulsion (Rauber \& Treue, 1998). Here, to eliminate the contamination from the reference repulsion, we used a discrimination task, rather than an estimation task, to measure the amount of motion repulsion, as both the reference stimulus and the test stimulus in the two-interval discrimination task would be repelled by the cardinal axis in a similar way (Chen et al., 2005; Rauber \& Treue, 1999).

\section{Procedure}

The main experiment consisted of pre-test sessions (2 days), training sessions (6 days), and post-test sessions (2 days) (Fig. 2A). In the pre-test session on day 1, the participants first practiced 20 supra-threshold trials to become familiarized with each task. Then, we used the method of constant stimuli to measure their baseline performance. For the main experiment and luminance training experiment (control experiment 1), we measured it using the direction discrimination task and the luminance discrimination task. For the speed training experiment (control experiment 2), the direction discrimination task and the speed discrimination were used. In the direction training experiment (control experiment 3), we measured it only with the direction discrimination task. No feedback was given to the participants. In the pre-test session on day 2 , the baseline performance was measured for the motion repulsion task with the method of constant stimuli.

During the training sessions, the participants were trained with separate tasks based on their assigned groups. In the main experiment, control experiment 1 , and control experiment 2 , we trained participants with the same set of stimulus parameters (luminance: $\varphi=127 \mathrm{gsv}$, speed: $4 \mathrm{deg} / \mathrm{s}$, direction: half of the participants trained with $\theta=30^{\circ}$ and the other half with $\theta$ $=-30^{\circ}$ ). For control experiment 3 , the participants were trained with $\theta=180^{\circ}, \varphi=127 \mathrm{gsv}$, and $4 \mathrm{deg} / \mathrm{s}$. The temporal 2AFC staircase procedure was used to equalize the participants' attentional engagement in each task. The classical 3down-1-up staircase rule was used, resulting in a $79.4 \%$ convergence accuracy level. Each staircase consisted of four preliminary reversals and six experimental reversals. The starting point for the direction discrimination task is $\theta \pm 10^{\circ}$, that for the luminance discrimination task is $\varphi \pm 40 \mathrm{gsv}$, and that for the speed discrimination is $4 \pm 0.9 \mathrm{deg} / \mathrm{s}$. The geometric mean of the experimental reversals was calculated as the threshold for each staircase run. There were 16 staircases in each session, which lasted for approximately $1 \mathrm{~h}$. Auditory feedback was given after incorrect responses.

The procedure for the post-test sessions was similar to that of the pre-test sessions, except that the measurement of the amount of motion repulsion in the post-test sessions was arranged on day 9 , which is before the measurement of the direction/luminance/speed task. This arrangement was set to ensure that the change in the motion repulsion can only be attributed to the training sessions, rather than the post-test sessions. The performance differences between the pre-test and post-test sessions were used to index the learning effect.

\section{Results}

\section{Main experiment: Direction discrimination training}

\section{Training effects}

After six days' training on the direction discrimination task, the participants' mean threshold of the first two staircases on each day decreased substantially over the course of the training (Fig. 3A). The best-fitting linear regression function was $y$ $=-0.40 \mathrm{x}+5.80$ with $\mathrm{R}^{2}=0.93$. The participants' performance on day 6 (threshold $=3.52^{\circ}, \mathrm{SEM}=0.25^{\circ}$ ) was significantly improved in comparison to that of day 1 (threshold $=$ $5.68^{\circ}, \mathrm{SEM}=0.44^{\circ}$; paired $\left.t(15)=5.13, p<0.001\right)$. We calculated the mean threshold of the first two staircases on each day to avoid contamination from the within-session learning (Hung \& Seitz, 2014). The results were similar when we calculated the mean threshold across all staircases. 

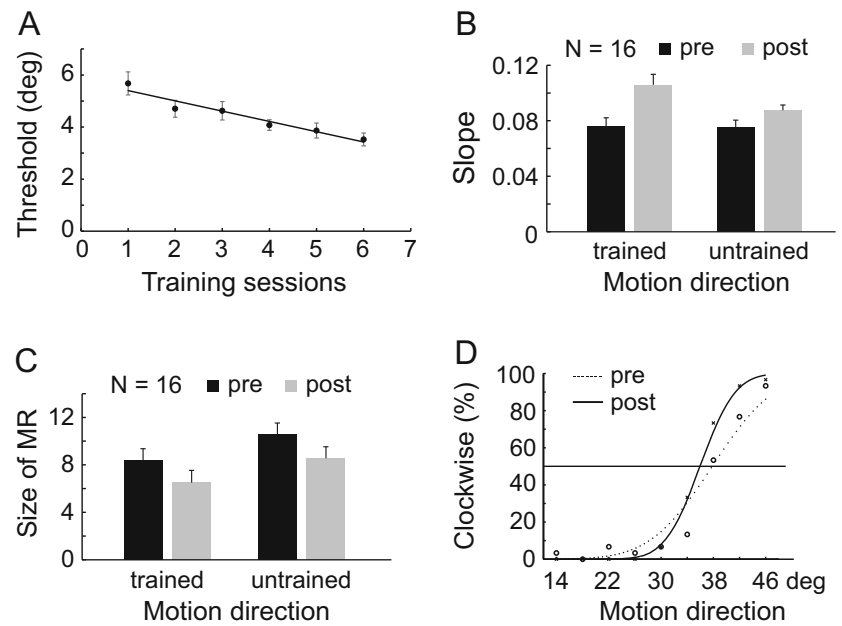

Fig. 3 Results of the main experiment. (A) The threshold of direction discrimination decreased substantially over the course of training. (B) Estimated direction discrimination sensitivity (slope) in the pre-test and post-test sessions. (C) Measured amount of motion repulsion in the pretest and post-test sessions. (D) Schematic illustration of the motion repulsion measurement based on the data from a single participant. The measurement was made before and after the training (trained direction: $30^{\circ}$; untrained direction: $-30^{\circ}$ ). Based on the recurrent model of perceptual learning, training was accompanied by a repulsive effect. Therefore, in the motion repulsion task, the test DRD along the directions larger than $30^{\circ}$ (e.g., $38^{\circ}$ or $46^{\circ}$ ) would be perceived more clockwise after the training, thus increasing the probability of a clockwise response and decreasing the amount of motion repulsion. However, similar effect on the direction smaller than $30^{\circ}$ (e.g., $14^{\circ}$ or $22^{\circ}$ ) has a subtle effect on the estimation of the point of subjective equality. Error bars represent standard errors of the mean across participants

To evaluate the specificity of the training effect, we measured the participants' performance in the direction discrimination task and the luminance discrimination task, both in the pre-test and post-test sessions. We fitted the participants' behavioral results with a cumulative Gaussian function and used the slope of the curve at the $50 \%$ percentile to index their sensitivities to the measured tasks. A repeated measures ANOVA on the slope of the direction discrimination task (motion direction (trained vs. untrained) $\times$ session (pre-test vs. post-test); Fig. 3B) revealed significant effects of the session $\left(F(1,15)=22.49, p<0.001, \eta_{\mathrm{p}}{ }^{2}=0.6\right)$ and its interaction with motion direction $\left(F(1,15)=5.52, p<0.05, \eta_{\mathrm{p}}^{2}=0.138\right)$, but not of the motion direction alone $(F(1,15)=2.40, p=0.14)$. Tests of the simple main effect revealed a larger slope for the trained direction than for the untrained direction in the post-test session $(F(1,15)=5.47, p<0.05)$, whereas no significant difference was observed in the pre-test session $(F(1,15)=0.02$, $p=0.88)$. These results suggest that the training on the direction discrimination task enhanced the participants' discrimination sensitivity, and this improvement was specific to the trained direction.

Additionally, we observed an improved performance in the luminance discrimination task after the direction discrimination training. A repeated measures ANOVA on the slope of the luminance discrimination task (motion direction (trained vs. untrained) $\times$ session (pre-test vs. post-test)) revealed significant effects for the session $(F(1,15)=30.52$, $\left.p<0.001, \eta_{\mathrm{p}}^{2}=0.67\right)$, but a significant effect was not observed for the motion direction $(F(1,15)=1.24, p=0.28)$ or their interaction $(F(1,15) \approx 0, p=0.99)$. The improvement in the sensitivity of the luminance discrimination task might be due to the test effect or the exposure in the training sessions.

\section{Direction discrimination training reduces the amount of motion repulsion}

For the motion repulsion task, we fitted the behavioral results with a cumulative Gaussian function to estimate the point of subjective equality (PSE) of the test DRD. The amount of motion repulsion was defined as the difference between the estimated PSE of the test DRD and the corresponding direction of the reference DRD. A repeated measures ANOVA (motion direction (trained vs. untrained) $\times$ session (pre-test vs. post-test); Fig. 3C) revealed a significant reduction in the amount of motion repulsion after training $(F(1,15)=18.75$, $\left.p=0.001, \eta_{\mathrm{p}}{ }^{2}=0.556\right)$. Neither the effect of the motion direction $(F(1,15)=2.43, p=0.14)$ nor its interaction with the session $(F(1,15)=0.01, p=0.91)$ reached a significant level.

As suggested by a recurrent model of perceptual learning (Teich \& Qian, 2003; see Fig. 5 for detailed description), training on a direction discrimination task reduces the responses of the neurons preferring the trained direction (Fig. 5, upper panel). In the motion repulsion task, when the transparent motion stimulus was presented, this neuronal reduction would weaken the competitiveness of the neurons preferring the trained direction, as the extent of the inhibition is assumed to be proportional to the initial response of these neurons (Wilson \& Kim, 1994). In this regard, the trained direction will be repulsed more by the untrained direction, whereas the untrained direction will be repulsed less by the trained direction. When the single motion stimulus interval of the motion repulsion task was presented (i.e., the test DRD), this reduced neural activity would also induce a repulsive effect on the test stimulus only when it is near the trained direction (Fig. 5, lower panel). As the amount of motion repulsion was defined as the point of subjective equality (PSE) of the two-interval discrimination task, this repulsive effect on the test stimulus would decrease the measured amount of motion repulsion along the trained direction (Fig. 3D). In all, based on this model, the measured motion repulsion in the current study along the trained direction is a net effect of two opponents, which is hard to predict in our behavioral study. In contrast, the model makes a clear prediction of a reduced amount of motion repulsion along the untrained direction after training, which was consistent with our results. Therefore, the change in the motion repulsion in the main 
experiment could be explained by the recurrent model of perceptual learning, and it provides new evidence for the changes in the sensory areas after perceptual training.

\section{Control experiment 1: Luminance discrimination training}

A previous study has shown that the amount of motion repulsion decreases with the increasing stimulus duration, indicating that adaptation may affect the inter-neuronal inhibition (Rauber \& Treue, 1999). During the training of the discrimination task, one motion direction was repeatedly exposed for approximately 6,000 times, so the behavioral effects observed in the main experiment may be a result of adaptation. To rule out this possible interpretation, we conducted a control experiment in which the direction discrimination training was replaced with luminance discrimination training.

The behavioral results from the training sessions showed that the participants' mean luminance discrimination threshold of the first two staircases on each day decreased substantially over the time course of the training. The best-fitting linear regression function was $y=-0.75 x+16.93$, with $\mathrm{R}^{2}=0.64$. The participants' threshold decreased significantly from day 1 (threshold $=16.58 \mathrm{gsv}, \mathrm{SEM}=0.99 \mathrm{gsv}$ ) to day 6 (threshold $=13.37$ gsv, SEM $=0.98$ gsv; paired $t(15)=2.93$, $p=0.01)$.

This training effect was also shown by the slope difference between the pre-test and post-test sessions. A repeated measures ANOVA (motion direction (trained vs. untrained) $\times$ session (pre-test vs. post-test)) revealed a significant effect of ses$\operatorname{sion}\left(F(1,15)=32.16, p<0.001, \eta_{\mathrm{p}}{ }^{2}=0.682\right)$, but no significant effect for motion direction $(F(1,15)=0.75, p=0.40)$ or its interaction with the session $(F(1,15)=0.32, p=0.58)$ was observed. These results suggest that training improved the sensitivity in the luminance discrimination task, but this improvement was not specific to the trained direction.

In addition, this training effect was transferred between tasks. A repeated measures ANOVA on the slope of the direction discrimination task (motion direction (trained vs. untrained) $\times$ session (pre-test vs. post-test)) revealed no main effects (motion direction: $F(1,15)=0.26, p=0.62$; session: $F(1,15)=4.01, p=0.06)$ but there was a significant interaction effect $\left(F(1,15)=6.02, p<0.05, \eta_{\mathrm{p}}{ }^{2}=0.286\right)$. Further tests of the simple main effects revealed that the slope for the trained direction was enhanced significantly after training $(F(1,15)=9.94, p<0.01)$, while no significant difference was observed for the untrained direction $(F(1,15)=0.33, p=0.57)$, indicating a transfer of learning specific to the trained direction.

Importantly, the luminance discrimination training did not change the amount of motion repulsion. A repeated measures ANOVA (motion direction (trained vs. untrained) $\times$ session (pre-test vs. post-test); Fig. 4A) revealed no significant effects for motion direction $(F(1,15)=1.69, p=0.21)$ or session $(F(1,15)=1.41, p=0.25)$. A trend of significance was obtained for only their interaction $(F(1,15)=4.11, p=0.06)$. These results oppose the argument that the change in the motion repulsion in the main experiment was a result of adaptation.

\section{Control experiment 2: Speed discrimination training}

Chen and colleagues found that the amount of motion repulsion decreased when the participants diverted their attention to one direction of the transparent stimulus (Chen et al., 2005). Although we did not manipulate the participants' attention in the present study, intensive training along a specific direction may change the attentional allocation when the participants performed the motion repulsion task in the post-test session. To determine whether the behavioral effects on the motion repulsion observed in the main experiment stemmed from the training-induced attentional bias, we conducted a second control experiment in which the direction discrimination training was replaced with speed discrimination training. The speed discrimination task assured that the participants allocated similar amounts of attentional resources to the motion information as in the main experiment. If the change in the motion repulsion resulted from the training-induced attentional bias, we would expect to see a similar change in the amount of motion repulsion when the participants were trained with the speed discrimination task.

During the training sessions, the participants' performance, defined by the mean speed discrimination threshold of the first two staircases in each session, decreased gradually. The bestfitting linear regression function was $y=-0.03 x+0.57$ with $\mathrm{R}^{2}=0.95$. The participants' threshold decreased significantly from day 1 (threshold $=0.55 \mathrm{deg} / \mathrm{s}, \mathrm{SEM}=0.04 \mathrm{deg} / \mathrm{s}$ ) to day $6($ threshold $=0.41 \mathrm{deg} / \mathrm{s}$, SEM $=0.04 \mathrm{deg} / \mathrm{s} ;$ paired $t(15)=$ $4.15, p=0.001)$.

As in control experiment 1, the training effect of the speed discrimination task was also not specific to the trained direction. A repeated measures ANOVA (motion direction (trained vs. untrained) $\times$ session (pre-test vs. post-test)) with the slopes estimated in the pre-test and post-test sessions also revealed a significant effect of session $(F(1,15)=45.57, p<0.001$, $\eta_{\mathrm{p}}{ }^{2}=0.752$ ), but no significant effect of motion direction $(F(1,15)=0.42, p=0.53)$ or its interaction with session $(F(1,15)=0.01, p=0.91)$ was observed.

Similar to control experiment 1 , we found a training-induced between-task transfer effect. A repeated measures ANOVA on the slope of the direction discrimination task (motion direction (trained vs. untrained) $\times$ session (pre-test vs. post-test)) revealed a significant effect of session $(F(1,15)=16.17, p=0.001$, $\left.\eta_{\mathrm{p}}{ }^{2}=0.519\right)$ and a marginally significant interaction effect with motion direction $\left(F(1,15)=4.49, p=0.051, \eta_{\mathrm{p}}{ }^{2}=0.23\right)$, but no main effect of motion direction $(F(1,15)=0.11 p=0.74)$ was observed. Further tests of the simple main effects showed an increased slope for the trained direction after training 


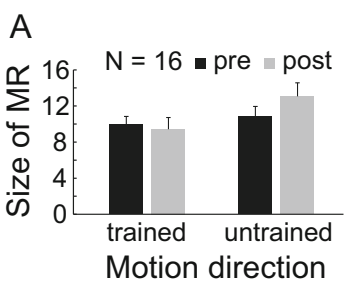

Fig. 4 Results of the control experiments. Measured amount of motion repulsion both before and after training with (A) the luminance discrimination task (control experiment 1), (B) the speed discrimination
B

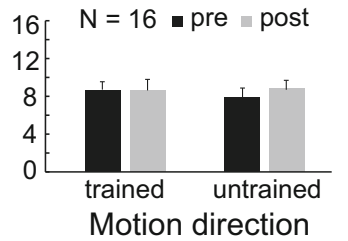

C

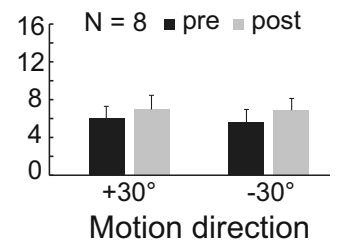

task (control experiment 2), and (C) the direction discrimination task at $150^{\circ}$ away (control experiment 3 ). Error bars represent standard errors of the mean across participants

$(F(1,15)=20.72, p<0.001)$, while no significant difference was observed for the untrained direction $(F(1,15)=4.05$, $p>0.05)$, indicating that the transfer of the learning effect was specific to the trained direction.

Importantly, speed discrimination training did not change the amount of motion repulsion. A repeated measures ANOVA (motion direction (trained vs. untrained) $\times$ session (pre-test vs. post-test); Fig. 4B) revealed no significant effects of motion direction $(F(1,15)=0.04, p=0.85)$, session $(F(1,15)=0.83, p=0.38)$, or their interaction $(F(1,15)=$ $0.82, p=0.38)$. These results, together with those of control experiment 1 , argue against the claim that the change in the amount of motion repulsion in the main experiment was a result of adaptation. These results also eliminate the alternative account of training-induced attentional bias in explaining the main experiment.

\section{Control experiment 3: Direction discrimination training}

Recently, Watanabe and his colleagues provided a new comprehensive framework of perceptual learning (Watanabe \& Sasaki, 2015). In their framework, the training effect reflects plastic changes in both feature- and task-related factors. This gives rise to another possible explanation for our major findings from the main experiment, namely that training-induced task learning contributes to the change in the motion repulsion. To eliminate this account, we conducted a direction discrimination training control experiment, in which we trained participants with a direction $150^{\circ}$ away from both directions in the transparent stimulus to determine whether it changes the amount of motion repulsion.

During the training sessions, the participants' performance, defined by the mean direction discrimination threshold of the first two staircases in each session, decreased gradually. The best-fitting linear regression function was $y=-0.17 x+3.51$, with $\mathrm{R}^{2}=0.64$. The participants' threshold decreased from day 1 (threshold $\left.=3.09^{\circ}, \mathrm{SEM}=0.38^{\circ}\right)$ to day 6 (threshold $=2.41^{\circ}, \mathrm{SEM}=0.31^{\circ}$; paired $\left.t(7)=2.35, p=0.051\right)$. The discrimination training along a direction $150^{\circ}$ away from the directions in the transparent stimulus did not change the motion repulsion. A repeated measures ANOVA (motion direction $\left(+30^{\circ}\right.$ vs. $\left.-30^{\circ}\right) \times$ session (pre-test vs. post-test); Fig. 4C) revealed no significant effects for the motion direction $(F(1,7)=0.02, p=0.90)$, session $(F(1,7)=2.41$, $p=0.16)$, or their interaction $(F(1,7)=0.10, p=0.76)$. These results suggest that task-based learning is not sufficient to induce the change in the motion repulsion observed in the main experiment.

\section{Discussion}

In the current study, we demonstrated that the amount of the perceived motion repulsion was reduced after perceptual training with the motion direction discrimination task. Combining the mutual inhibition model of motion repulsion and the recurrent model of perceptual learning, we can make a clear prediction of the change in the motion repulsion along the untrained direction, which was consistent with our behavioral results. The results from a series of control experiments eliminated the accounts of motion adaptation, attentional bias, and task learning in contributing to the reduction of the motion repulsion effect in the main experiment.

The mutual inhibition hypothesis is a prevailing theoretical interpretation of the motion repulsion phenomenon. This hypothesis could explain most of the behavioral findings (Blakemore \& Carpenter, 1970; Chen et al., 2005; Hiris \& Blake, 1996; Kim \& Wilson, 1996; Marshak \& Sekuler, 1979; Rauber \& Treue, 1999; Wilson \& Kim, 1994). Importantly, a computational model based on this hypothesis (Wilson \& Kim, 1994) has explained the change in the amount of motion repulsion across different direction separations. Previous physiological results have also demonstrated that MT neurons show firing patterns similar to those of mutual inhibition under transparent stimuli (Snowden, Treue, Erickson, \& Andersen, 1991; Treue, Hol, \& Rauber, 2000; Xiao \& Huang, 2015). Taken together, the mutual inhibition hypothesis may be the main mechanism underlying the motion repulsion phenomenon. In line with the prediction from the mutual inhibition model of motion repulsion and recurrent model of perceptual learning, the reduction of the motion repulsion along the untrained direction can be ascribed to the 
weakened inhibitory influences from the trained direction, which was presented at the same time and spatial location.

Importantly, the reduced amount of motion repulsion could not be explained by the decision-relation changes, such as the reweighting of the connections between the sensory and decision areas. First, the training effect was specific to the trained direction, which means that the connection for the untrained direction may remain unchanged and thus cannot explain the reduced amount of motion repulsion along the untrained direction. Second, based on the reweighting model of perceptual learning (Dosher, Jeter, Liu, \& Lu, 2013; Dosher \& Lu, 1999; Petrov, Dosher, \& Lu, 2005), the changed connections contributed to the enhanced slope, rather than the point of subject equality, of the psychophysical function. However, it is worth noting that although our results cannot be attributed to the plasticity in the decision-making process, these results do not conflict with the reweighting model of perceptual learning, as perceptual learning may modify both the sensory and decision-making process simultaneously.

A plausible explanation for the reduced amount of motion repulsion along the trained direction is related to the critical inferences from Teich and Qian's (2003) model on perceptual learning: the training on motion direction discrimination is accompanied by decreased activity for the neuronal population preferring the trained direction. In the motion repulsion task, the reduced neural activity would render the neurons preferring the trained direction less competitive and enhance the repulsion effect along the trained direction in the first stimulus interval (Fig. 1). This reduction would also shift the perceived direction (defined as the vector summation of the activity of all neurons) of the test stimulus in the second interval (Fig. 5). The shift effect on the test stimulus may exhibit a larger impact than the repulsion effect on the reference interval, leading to the reduced amount motion repulsion observed in the present study.

Our finding of the learning-induced modulation of the motion illusion seems contradictory to that of a previous study by Petrov and Van Horn (2012). In their results, motion learning exerted little impact on the motion after-effect duration, disagreeing with the representation modification hypothesis. Here, we provide explanations for the apparent contradictions between the two studies. First, we used the constant stimuli method and the forced choice task to measure the change in the motion repulsion. This approach is considered more objective and sensitive than their subjective report. Second, their study used different tasks in the training and test phases, which might attenuate the learning effect because the perceptual training effect was reserved only when similar tasks were used (Li, Piëch, \& Gilbert, 2004). Therefore, the absence of learning effects on the duration of the motion after-effect may
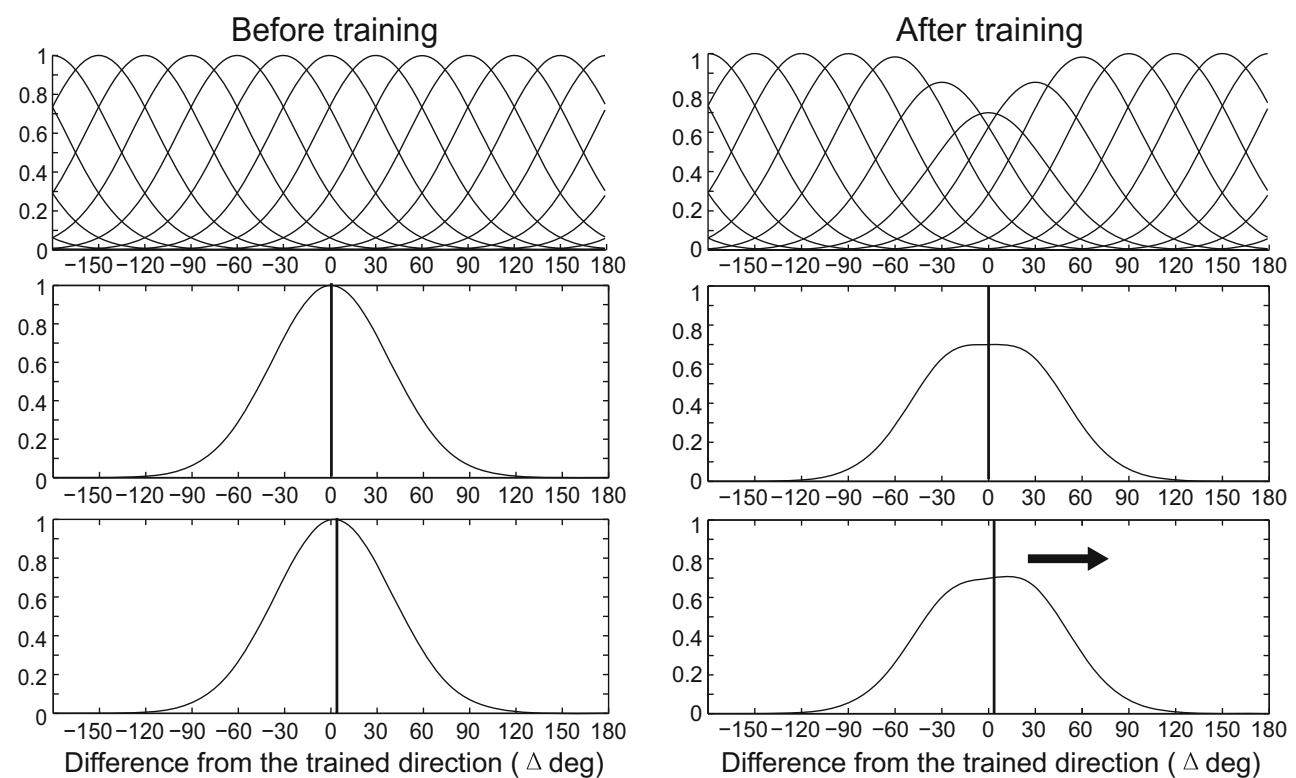

Fig. 5 The recurrent model of discrimination learning (Teich \& Qian, 2003). To measure the discrimination threshold both before and after training, the participants were asked to compare a reference direction (i.e., trained direction) to a test direction (a direction that is shifted by a small angle away from the trained direction). Upper panel: Tuning curves of the modelled neurons before and after training. The model assumes that the responses of neurons preferring the trained direction are reduced after training. Middle panel: Responses of all modelled neurons (preferred directions ranging from $-150^{\circ}$ to $180^{\circ}$ ) to the stimulus along the reference direction in the direction discrimination

task. The perceived direction was assumed to be the vector summation of these activities, which was also along the reference direction both before and after training. Lower panel: This panel mimics the responses of all modelled neurons to the test stimulus shifting $2^{\circ}$ away from the trained direction. The perceived direction (vector summation of the activities from all neurons) after training showed a repulsive effect, thus benefitting the behavioral performance. The vertical lines in the middle (i.e., along the trained direction) and lower panels (i.e., along a direction shifted $2^{\circ}$ away from the trained direction) represent the directions of the stimulus shown to the participants 
not be sufficient to speak against the representation modification hypothesis.

The training effect of the motion perceptual learning is suggested to be task-specific, even when similar stimuli are used across tasks (Saffell \& Matthews, 2003). However, in both the luminance discrimination and speed discrimination control experiments, we observed a between-task transfer of the learning effect. This apparent contradiction may be derived from the different task settings in the two studies. In Saffell and Matthews' study, they introduced a jitter to the reference stimulus in the test phase, whereas we used a fixed reference stimulus in the present study. Therefore, the exposure of the untrained feature during the training phase may facilitate the formation of the template for the reference stimulus, thus benefiting the performance of the untrained task. This explanation also indicates that the specific enhancement of the direction discrimination sensitivity in the main and control experiments may be engaged in different neural mechanisms, leading to different modulatory effects on the amount of motion repulsion.

In summary, our study demonstrates that training on direction discrimination can benefit the precise direction perception of the transparent stimulus. These results agree with predictions based on the mutual inhibition model of motion repulsion and the recurrent model of perceptual learning. Future investigations with neurophysiological measurements are required to elucidate the precise mechanism underlying the observed behavior effects.

Acknowledgments This work was supported by the National Natural Science Foundation of China (31230029, 31271081, 31470974), the National High Technology Research and Development Program of China (863 Program) (2012AA011602), and the Program for New Century Excellent Talents in University, State Education Ministry.

\section{References}

Ahissar, M., \& Hochstein, S. (1997). Task difficulty and the specificity of perceptual learning. Nature, 387, 401-406.

Ball, K., \& Sekuler, R. (1987). Direction-specific improvement in motion discrimination. Vision Research, 27(6), 953-965.

Blakemore, C., \& Carpenter, R. H. S. (1970). Lateral inhibition between orientation detectors in the human visual system. Nature, 228, 3739.

Brainard, D. H. (1997). The Psychophysics Toolbox. Spatial Vision, 10(4), 433-436.

Britten, K. H., Shadlen, M. N., Newsome, W. T., \& Movshon, J. A. (1992). The analysis of visual motion: A comparison of neuronal and psychophysical performance. The Journal of Neuroscience, 12(12), 4745-4765.

Byers, A., \& Serences, J. T. (2014). Enhanced attentional gain as a mechanism for generalized perceptual learning in human visual cortex. Journal of Neurophysiology, 112(5), 12171227. doi:10.1152/jn.00353.2014
Chen, Y., Meng, X., Matthews, N., \& Qian, N. (2005). Effects of attention on motion repulsion. Vision Research, 45(10), 1329-1339. doi:10.1016/j.visres.2004.11.005

Crist, R. E., Kapadia, M. K., Westheimer, G., \& Gilbert, C. D. (1997). Perceptual learning of spatial localization: Specificity for orientation, position, and context. Journal of Neurophysiology, 78, 28892894.

de Bruyn, B., \& Orban, G. A. (1988). Human velocity and direction discrimination measured with random dot patterns. Vision Research, 28(12), 1323-1335. doi:10.1016/0042-6989(88)90064-8

Dosher, B. A., \& Lu, Z. (1998). Perceptual learning reflects external noise filtering and internal noise reduction through channel reweighting. Proceedings of the National Academy of Sciences, 95, 13988 13993.

Dosher, B. A., \& Lu, Z.-L. (1999). Mechanisms of perceptual learning. Vision Research, 39, 3197-3221. doi: 10.1556/LP.1.2009.1.3

Dosher, B. A., Jeter, P., Liu, J., \& Lu, Z. (2013). An integrated reweighting theory of perceptual learning. Proceedings of the National Academy of Sciences, 110(33), 13678-13683. doi:10.1073/pnas. 1312552110

Fahle, M. (1997). Specificity of learning curvature, orientation, and vernier discriminations. Vision Research, 37(14), 1885-1895.

Fahle, M., \& Morgan, M. (1996). No transfer of perceptual learning between similar stimuli in the same retinal position. Current Biology, 6(3), 292-297.

Fine, I., \& Jacobs, R. A. (2002). Comparing perceptual learning across tasks: A review. Journal of Vision, 2, 190-203.

Fiorentini, A., \& Berardi, N. (1980). Perceptual learning specific for orientation and spatial frequency. Nature, 287, 43-44.

Gilbert, C. D., Sigman, M., \& Crist, R. E. (2001). The neural basis of perceptual learning. Neuron, 31, 681-697.

Hiris, E., \& Blake, R. (1996). Direction repulsion in motion transparency. Visual Neuroscience, 13(1), 187-197.

Hua, T., Bao, P., Huang, C.-B., Wang, Z., Xu, J., Zhou, Y., \& Lu, Z.-L. (2010). Perceptual learning improves contrast sensitivity of V1 neurons in cats. Current Biology, 20(10), 887-894. doi:10.1016/j. cub.2010.03.066

Hung, S., \& Seitz, A. R. (2014). Prolonged training at threshold promotes robust retinotopic specificity in perceptual learning. The Journal of Neuroscience, 34(25), 8423-8431. doi: 10.1523/JNEUROSCI.0745-14.2014

Jehee, J. F. M., Ling, S., Swisher, J. D., Van Bergen, R. S., \& Tong, F. (2012). Perceptual learning selectively refines orientation representations in Early Visual Cortex. The Journal of Neuroscience, 32(47), 16747-16753. doi:10.1523/JNEUROSCI.6112-11.2012

Karni, A., \& Sagi, D. (1991). Where practice makes perfect in texture discrimination: Evidence for primary visual cortex plasticity. Proceedings of the National Academy of Sciences, 88(11), 49664970.

Kim, J., \& Wilson, H. R. (1996). Direction repulsion between components in motion transparency. Vision Research, 36(8), 1177-1187. doi:10.1016/0042-6989(95)00153-0

Law, C.-T., \& Gold, J. I. (2008). Neural correlates of perceptual learning in a sensory-motor, but not a sensory, cortical area. Nature Neuroscience, 11(4), 505-513. doi:10.1038/nn2070

Law, C.-T., \& Gold, J. I. (2009). Reinforcement learning can account for associative and perceptual learning on a visual-decision task. Nature Neuroscience, 12(5), 655-663. doi:10.1038/nn.2304

Li, W., Piëch, V., \& Gilbert, C. D. (2004). Perceptual learning and topdown influences in primary visual cortex. Nature Neuroscience, 7(6), 651-657. doi:10.1038/nn1255

Marshak, W., \& Sekuler, R. (1979). Mutual repulsion between moving visual targets. Science, 205, 1399-1401.

Pelli, D. G. (1997). The VideoToolbox software for visual psychophysics: Transforming numbers into movies. Spatial Vision, 10(4), 437-442. 
Petrov, A. A., \& Van Horn, N. M. (2012). Motion aftereffect duration is not changed by perceptual learning: Evidence against the representation modification hypothesis. Vision Research, 61, 4-14. doi:10.1016/j.visres.2011.08.005

Petrov, A. A., Dosher, B. A., \& Lu, Z. (2005). The dynamics of perceptual learning: An incremental reweighting model. Psychological Review, 112(4), 715-743. doi:10.1037/0033-295X.112.4.715

Rauber, H. J., \& Treue, S. (1998). Reference repulsion when judging the direction of visual motion. Perception, 27(4), 393-402. doi:10.1068/p270393

Rauber, H. J., \& Treue, S. (1999). Revisiting motion repulsion: Evidence for a general phenomenon? Vision Research, 39(19), 3187-3196.

Recanzone, G. H., Merzenich, M. M., Jenkins, W. M., Grajski, K. A., \& Dinse, H. R. (1992). Topographic reorganization of the hand representation in cortical area $3 \mathrm{~b}$ owl monkeys trained in a frequencydiscrimination task. Journal of Neurophysiology, 67(5), 1031-1056.

Recanzone, G. H., Merzenich, M. M., \& Schreiner, C. E. (1992). Changes in the distributed temporal response properties of SI cortical neurons reflect improvements in performance on a temporally based tactile discrimination task. Journal of Neurophysiology, 67(5), 1071-1091.

Recanzone, G. H., Schreiner, C. E., \& Merzenich, M. M. (1993). Plasticity in the frequency representation of primary auditory cortex following discrimination training in adult owl monkeys. The Journal of Neuroscience, 13(1), 87-103.

Saffell, T., \& Matthews, N. (2003). Task-specific perceptual learning on speed and direction discrimination. Vision Research, 43(12), 13651374. doi:10.1016/S0042-6989(03)00137-8

Sagi, D., \& Tanne, D. (1994). Perceptual learning: Learning to see. Current Opinion in Neurobiology, 4, 195-199.
Schoups, A., Vogels, R., Qian, N., \& Orban, G. (2001). Practising orientation identification improves orientation coding in V1 neurons. Nature, 412, 549-553.

Shibata, K., Chang, L.-H., Kim, D., Nanez, J. E., Kamitani, Y., Watanabe, T., \& Sasaki, Y. (2012). Decoding reveals plasticity in V3A as a result of motion perceptual learning. PLoS ONE, 7(8), 1-7. doi:10.1371/journal.pone. 0044003

Shibata, K., Sagi, D., \& Watanabe, T. (2014). Two-stage model in perceptual learning: Toward a unified theory. Annals of the New York Academy of Sciences, 1316(1), 18-28. doi:10.1111/nyas.12419

Snowden, R. J., Treue, S., Erickson, R. G., \& Andersen, R. A. (1991). The response of area MT and V1 neurons to transparent motion. Journal of Neuroscience, 11(9), 2768-2785.

Teich, A. F., \& Qian, N. (2003). Learning and adaptation in a recurrent model of V1 orientation selectivity. Journal of Neurophysiology, 89(4), 2086-2100. doi:10.1152/jn.00970.2002

Treue, S., Hol, K., \& Rauber, H. J. (2000). Seeing multiple directions of motion-physiology and psychophysics. Nature Neuroscience, 3(3), 270-276. doi:10.1038/72985

Watanabe, T., \& Sasaki, Y. (2015). Perceptual learning: Toward a comprehensive theory. Annual Review of Psychology, 66(17), 1-25. doi:10.1146/annurev-psych-010814-015214

Wilson, H. R., \& Kim, J. (1994). A model for motion coherence and transparency. Visual Neuroscience, 11, 1205-1220.

Xiao, J., \& Huang, X. X. (2015). Distributed and dynamic neural encoding of multiple motion directions of transparently moving stimuli in cortical area MT. Journal of Neuroscience, 35(49), 16180-16198. doi:10.1523/JNEUROSCI.217515.2015 